\title{
Kauffman networks with threshold functions
}

\author{
Florian Greil and Barbara Drossel \\ Institut für Festkörperphysik, TU Darmstadt, Hochschulstraße 6, 64289 Darmstadt, Germany
}

(Dated: May 4, 2018)

\begin{abstract}
We investigate Threshold Random Boolean Networks with $K=2$ inputs per node, which are equivalent to Kauffman networks, with only part of the canalyzing functions as update functions. According to the simplest consideration these networks should be critical but it turns out that they show a rich variety of behaviors, including periodic and chaotic oscillations. The results are supported by analytical calculations and computer simulations.
\end{abstract}

Random Boolean networks (RBN) were introduced by S. Kauffman in 1969 [1, 2] to model the dynamics of genetic and metabolic networks [3] , but they are also used in a social and economic context [4, 5], and for neural networks. Although Boolean models represent a strong simplification of the far more complex reality, there exist several examples where the modelling of a genetic network by Boolean variables captures correctly the essential dynamics of the system [6, 7, 8 ]. For this reason, the study of RBNs remains an important step on the way towards understanding real networks.

A random Boolean network is a directed graph with randomly chosen links between $N$ binary nodes. We denote the state of a node with $\sigma_{i}= \pm 1$ (we call +1 "on" and -1 "off"), and the number of inputs per node with $K$. Each node $i$ is assigned at random an update function $f_{i}$. In this paper, we focus on the case $K=2$ and on threshold functions

$$
f_{i}=\operatorname{sign}\left(\sum_{j} \sigma_{j} c_{i j}\right) \equiv \operatorname{sign}\left(s_{i}\right)
$$

where the sum is taken over the two input nodes for node $i$, and $c_{i j}=-1$ for inhibitory connections and $c_{i j}=1$ for excitatory connections. (This version of RBN was used for instance in [9].) A connection is excitatory with probability $p_{+}$and inhibitory with probability $1-p_{+}$, leading to the following table (Tab. I).

\begin{tabular}{lllll}
\hline Input & $f_{7}$ & $f_{11}$ & $f_{13}$ & $f_{14}$ \\
\hline$(\downarrow, \downarrow)$ & $\uparrow$ & $\uparrow$ & $\uparrow$ & $\downarrow$ \\
$(\downarrow, \uparrow)$ & $\uparrow$ & $\uparrow$ & $\downarrow$ & $\uparrow$ \\
$(\uparrow, \downarrow)$ & $\uparrow$ & $\downarrow$ & $\uparrow$ & $\uparrow$ \\
$(\uparrow, \uparrow)$ & $\downarrow$ & $\uparrow$ & $\uparrow$ & $\uparrow$ \\
\hline probability & $\left(1-p_{+}\right)^{2}$ & $p_{+}\left(1-p_{+}\right)$ & $p_{+}\left(1-p_{+}\right)$ & $p_{+}^{2}$ \\
\hline
\end{tabular}

TABLE I: The four possible update functions for the model used in this paper. The input configuration is given in the first column, with $\uparrow$ denoting $\sigma_{i}=1$ and $\downarrow$ denoting $\sigma_{i}=-1$. The last row gives the probability for each function, the top row gives the name of the function according to the Kauffman model.

In agreement with other authors, we define $\operatorname{sign}(0)=1$. Threshold functions are used not only in the context of neural networks, but also in models for genetic networks [7, 9, 10]. These functions represent four of the 12 canalyzing update functions of Kauffman networks. Canalyzing functions are those non-frozen functions where at least one value of a given input can fix the output of a node, irrespective of the value of the second input. All nodes are updated in parallel according to the rule

$$
\sigma_{i}(t+1)=f_{i}\left(\left\{\sigma_{j}(t)\right\}\right) \equiv f_{i}\left(\sigma_{i_{1}}(t), \sigma_{i_{2}}(t)\right) .
$$

Node $i$ depends on the nodes $j$, namely on node $i_{1}$ and $i_{2}$.

The configuration of the system $\vec{\sigma} \equiv\left\{\sigma_{1}, \ldots, \sigma_{N}\right\}$ performs a trajectory in configuration space. As the state space is finite and the dynamics is discrete, some states will occur again. If a cycle in state space has a set of transient states leading to it, it is called an attractor.

Kauffman classified the dynamics of RBNs according to whether it is chaotic or frozen or critical (as described in the review [11]). In a chaotic network, a perturbation at one node propagates on an average to more than one node, leading to long attractors. In a frozen network, such a perturbation propagates on an average to less than one node, and in the thermodynamic limit $N \rightarrow \infty$ only a finite number of nodes are not constant after a certain transient time. Critical networks are at the boundary between these two types of behavior, with a perturbation of one node propagating on an average to one other node. Therefore the difference between two almost identical initial states increases like a power law in time. The number of nodes that are not frozen on all attractors increases in a critical network as a power law $\sim N^{2 / 3}$ of the system size $N$, as was found numerically in [12] and analytically in [13, 14].

It is the aim of this paper to show that this classification breaks down for the simple class of RBNs considered here. We find a much richer dynamical behavior with not only a frozen and a chaotic phase, but also with two types of oscillating phases and several critical points.

Let us first apply the criticality condition in its simplest version: For all four update functions, the probability that the output changes if one input spin is flipped, is $1 / 2$. Since each node is on an average the input to two other nodes, a perturbation at one node propagates on an average to one other node, and we should expect 
the model to be critical. This is in agreement with the finding that $K=2$-RBNs that contain only canalyzing update functions (but all of them with the same probability) are critical [15]. However, this simple argument is based on the assumption that all four possible input configurations occur equally often, which may be true at the beginning of a simulation run, but may be wrong already after one timestep. For this reason, Moreira and Amaral [16] argued that the calculation should be performed such that the input configurations are weighted with their frequencies in the stationary state.

Let us therefore next apply the rule given by Moreira and Amaral and let us determine for what values of $p_{+}$ it predicts that the model is frozen, critical, or chaotic. We will then see later that the result is still not correct for all parameter values.

We denote with $b_{t}$ the proportion of nodes in state $\sigma_{i}=$ +1 at time $t$. In the thermodynamic limit, it changes deterministically according to

$$
\begin{aligned}
b_{t+1}= & 1-\left[b_{t}^{2}\left(1-p_{+}\right)^{2}+\left(1-b_{t}\right)^{2} p_{+}^{2}+\right. \\
& \left.2 b_{t}\left(1-b_{t}\right) p_{+}\left(1-p_{+}\right)\right] .
\end{aligned}
$$

The expression in the square brackets is the probability that an input combination leads to $s_{i}=-2$. In the stationary state, we have $b_{t+1}=b_{t}=b$ with

$$
b\left(p_{+}\right)=\frac{4 p_{+}^{2}-2 p_{+}-1 \pm \sqrt{5-12 p_{+}+8 p_{+}^{2}}}{2\left(1-2 p_{+}\right)^{2}} .
$$

The sign in the numerator has to be chosen such that $b \in[0,1]$, therefore only the positive branch remains, see Fig. [1. For $p_{+}=1 / 2$, the denominator vanishes, and the stationary solution of Eq. (3) is $b=3 / 4$. For $p_{+}=1$, we have $b=0$ and $b=1$, with the first solution being obviously unstable as it is destroyed by one node in the state $\sigma_{i}=+1$. The second solution is a stable fixed point of the dynamics. For $p_{+}=0$, we have $b=(-1+\sqrt{5}) / 2$.

The mean number of nodes to which a perturbation at one node propagates is in the stationary state given by $2 \pi_{1}$, with $\pi_{1}$ being the probability that a node changes its state when one input is flipped. We obtain it by adding up the probabilities for those input configurations which allow a transition between an output +1 and -1 and vice versa. This is true for half of the input configurations leading to $s_{i}=0$ (the first 4 terms in the following equation) and for all input configurations for which $s_{i}=-2$ (the last 4 terms):

$$
\begin{aligned}
\pi_{1}= & \left(1-p_{+}\right)(1-b)\left(1-p_{+}\right) b+p_{+} b p_{+}(1-b)+ \\
& p_{+} b\left(1-p_{+}\right) b+\left(1-p_{+}\right)(1-b) p_{+}(1-b)+ \\
& \left(1-p_{+}\right) b\left(1-p_{+}\right) b+p_{+} b\left(1-p_{+}\right)(1-b)+ \\
& \left(1-p_{+}\right)(1-b) p_{+} b+p_{+}(1-b) p_{+}(1-b) \\
\Rightarrow \pi_{1}= & b+p_{+}-2 b p_{+}
\end{aligned}
$$

For $p_{+}=1 / 2$, we obtain $\pi_{1}=1 / 2$, for $p_{+}=1$, we obtain $\pi_{1}=0$. For $p_{+}=0$, we obtain $\pi_{1}=b \simeq 0.618$. We therefore conclude that the model is in the frozen phase for $p_{+}>1 / 2$, that it is critical for $p_{+}=1 / 2$, and chaotic for $p_{+}<1 / 2$.

The same result is obtained by calculating the stationary value of the Hamming distance between two identical network realizations. The Hamming distance is the fraction of nodes for two configurations $\vec{\sigma}, \tilde{\vec{\sigma}}$ that have different values: $D=(4 N)^{-1} \sum_{i=1}^{N}\left(\sigma_{i}-\tilde{\sigma}_{i}\right)^{2}$. If we denote with $\pi_{2}$ the probability that a node changes its state when both inputs are flipped, the time evolution of $D$ is given by

$$
D_{t+1}=2 D_{t}\left(1-D_{t}\right) \pi_{1}+D_{t}^{2} \pi_{2} .
$$

$\pi_{2}$ in the stationary state is obtained by summing all 8 combinations leading to $s_{i} \in\{ \pm 2\}$. It can be written as

$$
\pi_{2}=1-2 b\left(1-2 p_{+}\right)^{2}+2 b^{2}\left(1-2 p_{+}\right)^{2}-2 p_{+}+2 p_{+}^{2}
$$

and is $1 / 2$ for $p_{+}=1 / 2$ and 1 for $p_{+}=1$. If $D_{t}$ is very small, we have

$$
D_{t+1} \simeq 2 D_{t} \pi_{1},
$$

which allows for the growth of a small perturbation if $2 \pi_{1}>1$ or $p_{+}<1 / 2$, in agreement with our result above. The transition from a stationary value $D=0$ to a stationary value $D>0$ occurs at the same point.

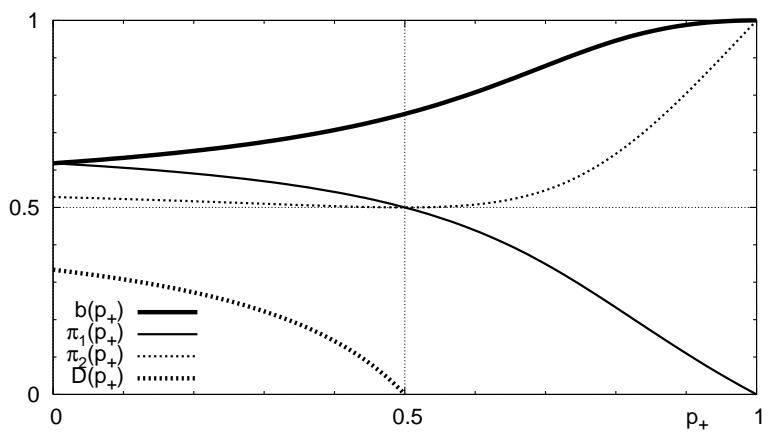

FIG. 1: The functions $b\left(p_{+}\right), \pi_{1}\left(p_{+}\right), \pi_{2}\left(p_{+}\right)$and the stationary value $D\left(p_{+}\right)$vs. $p_{+}$.

Fig. 2 2 shows $D_{t}$ for different values of $p_{+}$and for given initial conditions. One can see that $D_{t}$ approaches 0 for large times if $p_{+}>0.5$. Furthermore, one can see that $D_{t}$ oscillates with period 2 for the smaller values of $p_{+}$. This oscillation is an indication that the dynamics in the "chaotic" phase has some structure, which shall be investigated in the following.

Let us therefore have a closer look at the supposedly chaotic phase $p_{+}<1 / 2$. We will see that the dynamics is not chaotic at all for sufficiently small $p_{+}$. The considerations that have lead to our simple phase diagram are flawed. The reason is that we have assumed that $b$ becomes for large times stationary for all $p_{+}$. In order to see that this need not be the case, let us first look at 


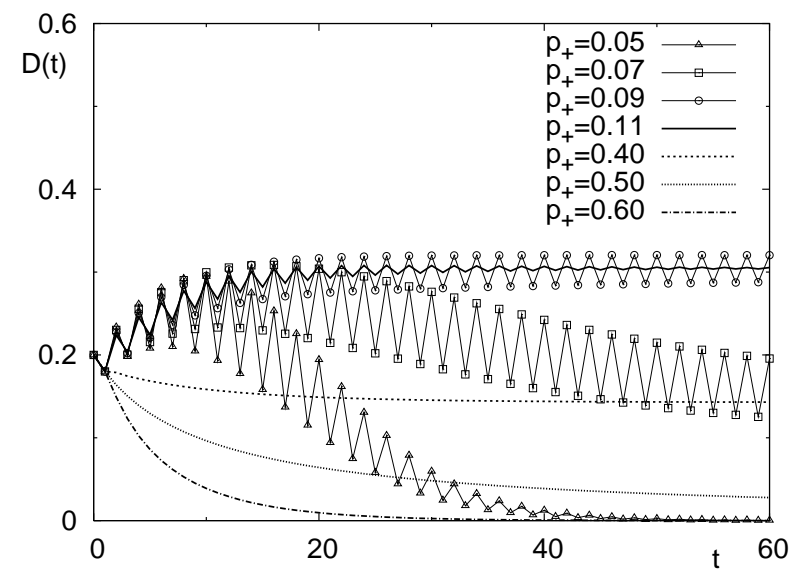

FIG. 2: The time evolution of the Hamming distance $D$ for different values of $p_{+}$when $b$ is not stationary. The curves are calculated according to Eqs. (3), (6) starting from $D_{0}=0.2$ and $b_{0}=0.5$.

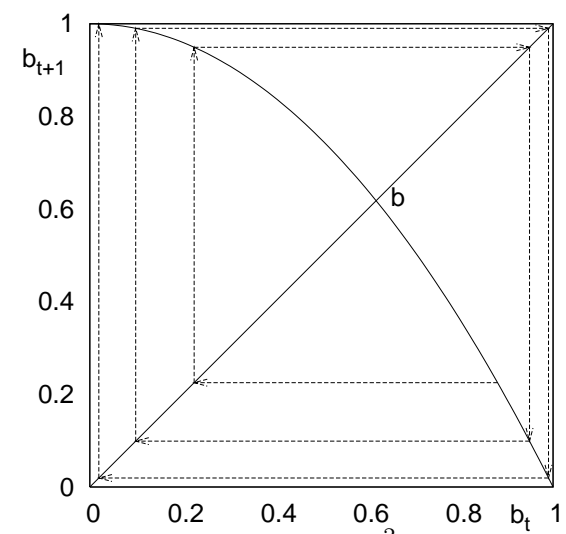

FIG. 3: The map $b_{t}$ vs. $b_{t+1}=1-b_{t}^{2}$ for $p_{+} \stackrel{b_{t}}{=} 0$. The fixed point $b$ is unstable as depicted by a sample trajectory.

the situation where $p_{+}=0$ : We then have $b_{t+1}=1-b_{t}^{2}$. This is a one-dimensional map shown in Fig. 3, The fixed point is unstable! Instead of having a stationary point with a constant proportion of nodes in the two states, the system oscillates between a configuration where all nodes are switched on and a state where all nodes are switched off. This is not chaotic dynamics at all, but very stable dynamics. In order to determine the range of $p_{+}$values, for which the fixed point value of $b$ is unstable, we performed a linear stability analysis. The ansatz $b_{t+1}\left(b+\delta b_{t}\right)=b+\delta b_{t+1}$ leads in linear order in $\delta b$ and for $p_{+}<1 / 2$ to

$$
\begin{aligned}
\delta b_{t+1} & =-2\left(b_{t}\left(1-2 p_{+}\right)^{2}+\left(1-2 p_{+}\right) p_{+}\right) \delta b_{t} \\
& =\left(1-\sqrt{5-12 p_{+}+8 p_{+}^{2}}\right) \delta b_{t}=: M \cdot \delta b_{t}
\end{aligned}
$$

In the last step we used Eq. (4). The fixed point is stable if the real part of $M$ is smaller than 1, which is the case if

$$
p_{+}>(3-\sqrt{7}) / 4 \equiv p_{c b} \approx 0.0886 .
$$

Only above this value does the system have a stationary state with constant proportions of nodes being "on" and "off".

We finally investigate in more detail the region $p_{+}<$ $p_{c b}$, where the proportion of "on" and "off" nodes oscillates with period 2. For $p_{+}=0$, every node oscillates with period 2, and we have a global attractor of period 2 . This need not necessarily be the case if $b$ oscillates with period 2 . The attractor could be much larger, while the proportion of off and on nodes oscillates still with period two. In order to determine for which parameters an attractor with period 2 is stable, we performed again a linear stability analysis, but now for two time steps together. We assume that the system is on an attractor of length 2. Let there be every even time step a proportion $x$ of "on"-nodes and every odd step a proportion $y$. The time evolution is given by Eq. (3), but now we combine two consecutive time steps. We flip one node and look how the Hamming distance grows in comparison to the undisturbed system after two time steps. The condition that information spreading is critical is in the cycle with period 2

$$
\pi_{1}(x) \cdot \pi_{1}(y)=\frac{1}{4}
$$

Combining Eq. (11) with the time evolution of $x$ and $y$ we obtain three equations

$$
\begin{aligned}
& y=1-\left[x^{2}\left(1-p_{+}\right)^{2}+(1-x)^{2} p_{+}^{2}+2 x(1-x) p_{+}\left(1-p_{+}\right)\right] \\
& x=1-\left[y^{2}\left(1-p_{+}\right)^{2}+(1-y)^{2} p_{+}^{2}+2 y(1-y) p_{+}\left(1-p_{+}\right)\right] \\
& \frac{1}{4}=\left(x+p_{+}-2 x p_{+}\right)\left(y+p_{+}-2 y p_{+}\right)
\end{aligned}
$$

This system can be solved numerically and we obtain a critical value $p_{c n}=0.0657$. For $p_{+}$below this value a perturbation at one node will die out and all nodes will again blink with period 2. Above this value, attractors must be longer than 2 .

We checked these analytical predictions by performing computer simulations. In order to identify the transition at $p_{c n}$, we measured the median attractor length.

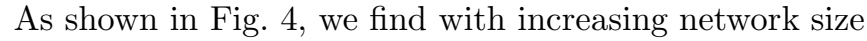
an increasingly sharp transition. Below the transition at $p_{c n}$, the proportion of attractors of length 2 converges to some nonzero value with increasing system size, indicating that cycles of length 2 are stable. Above the transition, the median attractor length increases more and more rapidly with increasing system size, indicating a diverging median. Another finding is that attractors become again shorter as the critical point $p_{+}=1 / 2$ is approached.

We also evaluated the frequency of phase jumps in $b(t)$ on the attractors. Each phase jump is a deviation from 


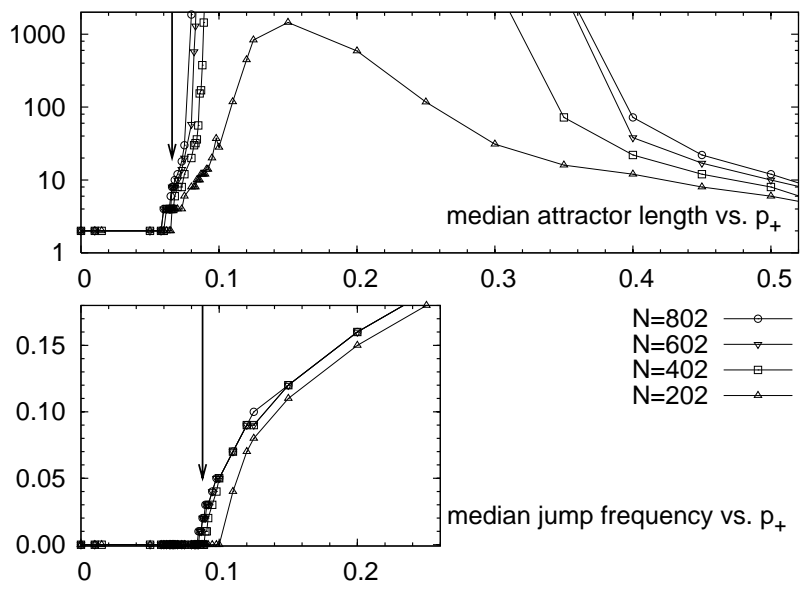

FIG. 4: Numerical verification for the transition $p_{c n}$ and $p_{c b}$, both marked by vertical arrows. The upper panel shows the median attractor length, the lower panel the median jump frequency on each attractor candidate in dependence of $p_{+}$. Each data point corresponds to 5000 sample networks of size $N$ with fixed $p_{+}$and two initial conditions per realization. The time evolution is limited to 5000 computational steps for both the transient and the attractor length. The median is therefore a more sensible observable than the mean value: If a attractor candidate cannot be verified because the time limit was already reached we can still calculate the median.

an oscillation with period 2. The result is shown in the lower part of Fig. 4. With increasing system size, there is an increasingly sharp transition at $p_{c b}$ between zero phase jumps and a finite proportion of phase jumps.

We summarize the different types of dynamical behavior in the following diagram, Fig. 5

\begin{tabular}{|l|l|l|}
\hline \multicolumn{2}{|l|}{ nonfrozen } & frozen \\
\hline$b$ has period 2 & $b$ is constant in time \\
\hline $\begin{array}{l}\text { all nodes have } \\
\text { period 2 }\end{array}$ & nodes oscillate differently or not at all \\
\hline$p_{+}$ & $p_{c n}$ & $p_{c b}$
\end{tabular}

FIG. 5: Overview of the dynamic behavior of the model with $K=2$ in dependence of the parameter $p_{+}$.

To conclude, we have shown that the simplest Threshold Random Boolean Network shows three different types of phase transitions and not just the generally expected transition between a frozen and a chaotic phase. For parameter values $p_{+}<p_{c n}$, all nodes oscillate stably with period two. For $p_{c n}<p_{+}<p_{c b}$, the fraction of on-nodes oscillate with period two, but attractors are longer. For $p_{c b}<p_{+}<1 / 2$, the dynamical behavior is chaotic in the sense defined by Kauffman. For $p_{+}>1 / 2$, the network is in the frozen phase.

The lesson to be learned from this study is that the dynamical behavior of Boolean networks can be much richer than expected from simple considerations. There is indeed no reason why some model should not also show global oscillations with higher periods or period doubling cascades in the temporal behavior of $b(t)$. Real genetic networks can be expected to have an even richer dynamical behavior. If the simple classification into "frozen", "critical" and "chaotic" networks fails already in the random model presented in this paper, it will be even less suitable for real genetic networks, which have attractors with very specific properties related to the function of the network. A more sophisticated way of describing and classifying the dynamical behavior of Boolean networks is therefore required.

This work was supported by the Deutsche Forschungsgemeinschaft (DFG) under Contract No. Dr200/4-1.

[1] S. A. Kauffman, J. Theo. Bio. 22, 437 (1969).

[2] S. Kauffman, Nature 224, 177 (1969).

[3] S. Kauffman, C. Peterson, B. Samuelsson, and C. Troein, in Proceedings of the National Academy of Sciences USA (2003), no. 25 in 100, pp. 14796-14799.

[4] J. M. Alexander, in Philosophy of Science Assoc. 18th Biennial Mtg (2002), URL http://philsci-archive.pitt.edu/archive/00001049/

[5] M. Paczuski, K. E. Bassler, and A. Corral, Phys. Rev. Lett. 84, 3185 (2000).

[6] S. Bornholdt, Science 310, 449 (2005).

[7] F. Li, T. Long, Y. Lu, Q. Ouyang, and C. Tang, Proc. Nat. Acad. Sci. 101, 4781 (2004).

[8] R. Albert and H. G. Othmer, Journal of Theoretical Biology 223, 1 (2003).

[9] T. Rohlf and S. Bornholdt, Physica A 310, 245 (2002).

[10] S. Bornholdt and K. Sneppen, Proc. R. Soc. London B 267, 2281 (2000).

[11] M. Aldana-Gonzalez, S. Coppersmith, and L. P. Kadanoff, Perspectives and Problems in Nonlinear Science pp. 23-89 (2003).

[12] J. E. S. Socolar and S. A. Kauffman, Phys. Rev. Lett. 90, 068702 (2003).

[13] B. Samuelsson and C. Troein, Phys. Rev. Lett. 90, 098701 (2003).

[14] V. Kaufman, T. Mihaljev, and B. Drossel, Phys. Rev. E 72, 046124 (2005).

[15] U. Paul, V. Kaufman, and B. Drossel, Phys. Rev. E 73, 026118 (2006).

[16] A. A. Moreira and L. A. N. Amaral, Phys. Rev. Lett. 94, 218702 (2005). 